\section{Airway management in adult epiglottitis}

\section{To the Editor:}

The management plan of acute epiglottitis in children is generally agreed upon.' It calls for airway control by tracheal intubation following mask induction of general anaesthesia using halothane and oxygen. Guidelines for the management of acute epiglottitis in adults, on the other hand, are less well defined. A protocol involving tracheal intubation following inhalational induction of general anaesthesia has been recommended. ${ }^{2}$ I would like to describe a case of adult epiglottitis that was managed according to that protocol, but not without difficulty.

A $41 \mathrm{yr}$, well-developed man presented with acute respiratory distress. He was sitting upright, drooling, and had an audible inspiratory stridor. His upper airway seemed adequate otherwise. A lateral radiograph of the neck was diagnostic of acute epiglottitis.

The patient was taken to the OR to provide an artificial airway by oral tracheal intubation. Following $i v$ glycopyrrolate, inhalational induction was started using a low concentration of halothane in $100 \%$ oxygen. The halothane concentration was gradually increased. When the patient started to lose consciousness, his respiratory efforts were gently assisted. Airway obstruction worsened as the depth of anaesthesia increased and finally became complete, despite chin lifting and forceful jaw thrusting. Direct laryngoscopy was attempted and tracheal intubation was achieved with difficulty.

When considering a management plan for this case, it was felt that direct laryngoscopy or fibreoptic intubation would have been poorly tolerated by the already distressed patient. Manipulation of the irritable upper airway also runs the risk of precipitating laryngeal spasm. Sedating patients with compromised airways could increase the obstruction or precipitate apnoea. In the meantime, anaesthetizing the larynx might increase the risk of pulmonary aspiration in the presence of severe distress, sympathetic stimulation and delayed gastric emptying. Intravenous induction of anaesthesia and the use of muscle relaxants were ruled out for fear of precipitating complete airway obstruction without the ability to intubate the trachea or ventilate the lungs.

Based on these considerations, inhalational induction was chosen. This case exemplifies its major risk under these circumstances - acute airway obstruction when consciousness is lost. Inhalational induction can also be more difficult in adults than in children due to the longer induction time and the stronger mandibular muscles in the adult. The favourable record of inhalational induction in children with epiglottitis may not, therefore, imply that it is as suitable for adults.

All the alternative approaches that could be employed for the airway management of adult epiglottitis might also be associated with difficulties. Thus, facilities to perform transtracheal jet ventilation and emergency tracheostomy should be available immediately. To a considerable extent, the choice of technique will depend on the clinician's skill and degree of comfort with the options available.

\section{Hannalah MD FFARCS \\ Department of Anesthesia \\ Georgetown University Research Center \\ Washington, DC.}

\section{REFERENCES}

1 Crockell DM, McGill TJ, Healy GB, Friedman EM. Airway management of acute supraglottitis at the children's hospital, Boston: 1980-1985. Ann Otol Rhinol Laryngol 1988; 97: 114-9.

2 Warner JA, Finlay WE. Fulminating epiglottitis in adults. Anesthesia 1985; 40: 348-52.

\section{Negative pressure pulmonary oedema}

\section{To the Editor:}

We read with interest the report of negative pressure pulmonary oedema in two awake infants given muscle relaxants. ${ }^{3}$ We agree with the authors that airway management during induction of general anaesthesia in the infant less than two months of age "requires a high level of experience and skill." We do not agree with their recommended practice of achieving airway control by giving non-depolarising muscle relaxants to awake infants.

It is a fundamental principle of any anaesthetic that before a muscle relaxant is given the anaesthetist should be able to maintain the patient's airway. In infants of this age, with an indwelling intravenous cannula, anaesthesia can be safely induced either by the intravenous or the inhalational route.

In either case, the airway should be clear and maintained by the anaesthetist before muscle relaxants are given.

In neither case was the infant anaesthetized before the administration of the muscle relaxant. In the first case, the infant received $0.25 \%$ halothane in $50 \% \quad \mathrm{~N}_{2} \mathrm{O} / \mathrm{O}_{2}$. The second case received $100 \% \mathrm{O}_{2}$. To say that the slower onset of non-depolarising drugs permits their earlier administration "so that maximal paralysis is present when 
a light surgical plane of anaesthesia has been reached" shows a poor grasp of their pharmacology. Muscle relaxants do not wait $120 \mathrm{sec}$ before working. There is a gradual onset of muscle relaxation which reaches its maximum at around $120 \mathrm{sec}$ for vecuronium. To give an awake infant this muscle relaxant and expect him to breathe sufficiently to deepen anaesthesia prior to intubation is unrealistic.

We routinely induce anaesthesia with an inhalational agent, such as halothane in $50 \% \mathrm{O}_{2} / \mathrm{N}_{2} \mathrm{O}$ and know that it takes several minutes to achieve surgical anaesthesia.

We would expect inhalational induction to be impossible with the infant partially paralysed as he would not be able to breathe enough to inhale the anaesthetic.

So we can assume that the infants are being paralysed and intubated whilst awake. We would consider this ethically unacceptable as alternative methods of inducing and maintaining anaesthesia safely are available.

U. Hodges BScHons MBBS FCAnaes

E. Sumner MA BM BCh FFARCS

Anaesthetic Department

Great Ormond Street Hospital

Great Ormond Street

London WCIN 3JH

England.

\section{REFERENCE}

I Warner LO, Martino JD, Davidson PJ, Beach, TP. Negative pressure pulmonary oedema: a potential hazard of muscle relaxants in awake infants. Can J. Anaesth 1990 , 37: 580-3.

\section{REPLY}

Thank you for the opportunity to respond to the letter by Drs. Hodges and Sumner, which is, we hope, an isolated case of misinterpretation of the modified inhalational induction technique utilized in our case report on negative pressure oedema referred to above.

It was not our intent to recommend the sequence of administering non-depolarizing inuscle relarants to awake infants, but merely to point out a potential serious problem (note to word "hazard" in the title) associated with it. Our discussion recounted most induction techniques in common use and stated that "an inhalational technique with gradually increasing concentrations of gaseous agents is preferred at our institu. tion." The administration of non-depolarizing muscle relarants at the beginning of an inhalational induction rather than after anaesthesia has been partially or lotally induced is simply one variation, the object being to "facilitate control of the airway and ventilation during the early stages of induction" by allowing the insertion of an oral airway earlier than could otherwise be accomplished. At this time, gentle assistance of respiration can either be assumed or contimued, and respiration controlled as paralysis sets in. (Obviously, a partially paralyzed infant will need assistance in breathing!) When a light surgical plane of anaesthesia has been reached, intubating conditions should be favourable. If the technique is properly executed, the infants are not "being paralysed and intubated whilst awake." In our two cases, problems arose because of lack of recognition and correction of airway obstruction. As the different onset times of diaphragmatic paralysis and paralysis of muscles protecting the upper airway were not well-described we did not anticipate an airway problem developing within seconds after the administration of a non-depolarizing relarant.

Aclually, the above-described techmique is seldom used at our institution, since most infonts either do not presemt with an intravenous line in place, or are at risk for gastric aspiration, and thus are not candidates for this approach. As physicians in a teaching hospital, we feel an obligation to expose trainees to a variety of induction techiniques.

It is unfortunate that our London colleagues chose to criticize an induction technique that they did not fully comprehend, while ignoring the broader educational aspects of the discussion.

Louise O. Warner MD

Department of Anesthesiology

Children's Hospital

700 Children's Drive

Columbus $\mathrm{OH} 43205$

\section{TURP syndrome}

To the Editor:

I was pleased to read the excellent review article on TUR syndrome by Dr. Jensen in the Journal (Can J Anaesth 1991: 38: 90-7).

I would like to know if Dr. Jensen encountered any patients exhibiting aberrant symptoms during TUR. Over the past six years I have seen six patients who had TURP under tetracaine spinal with small doses of diazepam for sedation. Twenty minutes after start of resection the patients complained of "burning in the ears" (two also complained of burning on the face). They had no other complaints. I discussed this with the surgeon and he said that he had heard similar complaints which went on to a full blown TUR syndrome.

Blood samples were drawn and analyzed in two of the patients and were found to have serum sodium concentration of $120 \mathrm{mM} \cdot \mathrm{L}^{-1}$. All the patients were treated with 20 $\mathrm{mg}$ of jurosemide. There were no further complications

Chidambaram Ananthanarayan MD FRCPC

University of Toronto

Department of Anaesthesia

Mount Sinai Hospital

\section{$R E P L Y$}

I wish to thank Dr. Allanthanarayan for introducing the topic of aberrant symptoms during transurethral resection 